To help assess the accuracy of data, NCATSIS has a comprehensive publication Assessing the Quality of Identification of Aboriginal and Torres Strait Islander People in Hospital Data, which includes guidelines for conducting a data quality audit. Procedures involve reinterviewing patients and comparing these results with the information in hospital records.

To assist in other ways the ABS provides related services through its State regional offices, including:

- providing expert advice on effective data capture;

- providing advice on train-the-trainer services for data collectors on how to collect indigenous data, or assisting organisations to adapt this training to suit local requirements;

- providing indigenous status awareness material;

- providing advice and assistance in conducting data quality assessments and assistance with analysis of results. 倍

For further information please contact Janis Shaw, NCATSIS 1800633216 , or by email at:

janice.shaw@abs.gov.au.

A list of all ABS publications is available on the ABS

Web site at www.abs.gov.au.

\title{
IMPROVING THE IDENTIFICATION OF ABORIGINAL AND TORRES STRAIT ISLANDER PEOPLES IN HEALTH-RELATED INFORMATION COLLECTION SYSTEMS IN NSW
}

\author{
Heather Simon \\ Contract and Service Performance Branch \\ NSW Department of Health \\ Angela Todd \\ Effective HealthCare Australia \\ University of Sydney
}

This article describes two projects undertaken by the NSW Department of Health to improve the identification of Aboriginal and Torres Strait Islander people in our major health information collection systems. The first project described is the development of Better Practice Guidelines for identification, and the second is a training program for frontline staff to improve the accuracy of patient registration information that is collected.

\section{BACKGROUND}

The poor health status of Aboriginal and Torres Strait Islander peoples is well documented. Aboriginal infants have lower birth-weights, and higher rates of stillbirth, neonatal and postnatal death compared with nonAboriginal infants. Aboriginal mothers represent nearly 30 per cent of all maternal deaths but comprise less than three per cent of all deliveries. Chronic diseases are more common among Aboriginals and Torres Strait Islanders, particularly diabetes, renal failure, and eye and ear problems. Aboriginal and Torres Strait Islander peoples are at significantly increased risk of death due to circulatory diseases, respiratory illnesses, injuries and poisoning, and cancer when compared with other Australians. The average estimated life expectancy of Aboriginals and Torres Strait Islanders continues to be around 15-20 years below that of non-indigenous
Australians, with estimates of 57 years for indigenous males and 62 years for indigenous females. ${ }^{1,2,3}$

Accurate and reliable information is critical to our efforts to improve the health outcomes of Aboriginal and Torres Strait Islander peoples. However, the quality of much of the available information is poor. One of the most important reasons for this is the under-identification of Aboriginal and Torres Strait Islander peoples in most health-related information collection systems. For example, it has been estimated that the NSW Hospital Inpatient Statistics Collection (ISC) under-enumerates Aboriginals and Torres Strait Islanders by 33 per cent. ${ }^{4}$ This problem is further compounded by inconsistent collection practices when the data are collected, in particular the use of various questions about Aboriginal origin, descent and identification.

The inaccuracy and unreliability of these data seriously affect their use for planning, evaluation and monitoring purposes at local, State and national levels. In this regard it is of particular concern that 'national' reports about Aboriginal and Torres Strait Islander health often do not include NSW health statistics due to problems with data quality. As a result, the 25 per cent of Aboriginal Australians who reside in NSW are not being represented in the national picture.

\section{BETTER PRACTICE GUIDELINES}

The NSW Department of Health's Contract and Service Performance Branch has initiated a number of projects aimed at identifying and supporting better practice within the NSW health system. One of these projects was the development of Better Practice Guidelines to improve the level of identification of Aboriginal and Torres Strait 
Islander people in the New South Wales public health system. This project was auspiced by the State Continuous Improvement Steering Committee and commenced in 1998.

The development of better practice guidelines draws on the process of 'benchmarking'. This form of quality improvement compares and contrasts the way a number of organisations perform the same function. The aim is to establish best practice, and then to introduce this practice into the other organisations.

Best practice in relation to the identification of Aboriginal and Torres Strait Islander peoples in major health data collections was established at a national meeting in November 1996. At this meeting it was agreed that the benchmark to collect information about Aboriginality would be the question and responses used by the Australian Bureau of Statistics (ABS) in the five-yearly population census (see Box above).

\author{
Are you of Aboriginal orTorres Strait islander origin? \\ For persons of both Aboriginal and Torres Strait \\ Islander origin, mark both 'Yes' boxes. \\ (I) No \\ Y Yes, Aboriginal \\ Y Yes, Torres Strait Islander
}

A survey of the current collection practices within the NSW health services identified a wide variation in the questions used to collect information describing Aboriginality or indigenous status. This finding led to the publication of a document entitled Better Practice Guidelines to improve the level of Aboriginal and Torres Strait Islander identification in the New South Wales public health system. ${ }^{5}$ The main aim of this document is to assist health services in addressing the changes needed in their current practices to meet the above benchmark.

The publication of the Better Practice Guidelines is one strategy used to introduce change. Increasing the awareness among staff of the importance of collecting high quality data and understanding the barriers to data collection of this nature has also been targeted, as described in the second project, the development of a training program for frontline staff collecting patient registration information.

\section{TRAINING PROGRAM FOR FRONTLINE STAFF}

In 1999, the Aboriginal Health Information Strategy (AHIS) Unit developed a draft training program for frontline staff to raise the awareness about, and to improve the quality of, Aboriginal and Torres Strait Islander origin information collected in NSW hospitals. Frontline staff are clerical or clinical staff who collect patient/client registration information at the first point of contact with health services, including:

- admission or booking offices

- emergency department

- community health centres

- ambulance services.

The draft program was piloted with over 100 frontline staff at five large public hospitals in NSW. A report of the pilot study was released in September $1999,{ }^{6}$ and is available from the AHIS Unit and from the AHIS intranet Web site at: internal.health.nsw.gov.au/iasd/imcs/ahisu/ publications.

The subsequent training program addresses the broader area of patient registration information, and the difficulties that can arise collecting various personal details. The program also covers issues specific to the collection of Aboriginal and Torres Strait Islander origin information. The training is being delivered statewide using two approaches:

- delivery of the program by AHIS staff to 'target' hospitals with significant numbers of Aboriginal people in their catchment area;

- training of health staff who can deliver the training program to all other public hospitals.

To supplement this work the AHIS Unit has also recently produced a pamphlet for use by all NSW Health staff to raise their awareness about the importance of accurately collecting and recording information describing the origin of Aboriginal and Torres Strait Islander people. ${ }^{7}$

\section{CONCLUSION}

It must be recognised that, despite these strategies, there are a number of factors that are likely to affect efforts to improve the quality of Aboriginal and Torres Strait Islander origin and other patient registration information. These include:

- effectively engaging people at different levels within the public health system;

- communicating with, and gaining the support of external stakeholders, in particular Aboriginal and Torres Strait Islander organisations and medical services;

- acknowledging the existence of negative attitudes towards Aboriginal and Torres Strait Islander people and how these affect their experience of the public health system, and consequently the information they provide;

- recognising the work pressures of frontline staff and providing them with better support and training;

- developing a stronger information culture that values and uses accurate health information. 
These issues present many challenges for the public health workforce in NSW.

\section{REFERENCES}

1. Evaluation Committee. The National Aboriginal Health Strategy: an evaluation. Sydney: NSW Department of Health, 1994.

2. Australian Bureau of Statistics. The Health and Welfare of Australia's Aboriginal and Torres Strait Islander Peoples. Canberra: AGPS, 1997.

3. Australian Bureau of Statistics. The Health and Welfare of Australia's Aboriginal and Torres Strait Islander Peoples. Canberra: AGPS, 1999.

4. Deeble J, Mathers C, Smith L, Goss J, Webb R and Smith V. Expenditures on Health Services for Aboriginal and Torres Strait Islander People. Canberra: AGPS, 1998. AIHW Catalogue No. HWE 6.
5. NSW Department of Health. Better Practice Guidelines to Improve the Level of Aboriginal and Torres Strait Islander Identification in the NSW Public Health System. Sydney: NSW Department of Health, 2000. State Health Publication No. CSP 980163.

6. NSW Department of Health. Improving Aboriginal and Torres Strait Islander origin information in NSW: Report of a pilot study. Sydney: NSW Department of Health, 2000. State Health Publication No. IMCS 990181.

7. NSW Department of Health. Aboriginal and Torres Strait Islander origin information: an important message for NSW Health Staff (pamphlet). Sydney: NSW Department of Health, 2000. State Health Publication No. IMCS 000059 .

For further information and inquiries regarding the Better Practice Guidelines contact Heather Simon, Contract and Service Performance Branch, NSW Department of Health by phone at (02) 93919434 , or by email at hsimon@doh.health.nsw.gov.au. For the training program and pamphlets contact Peter Williams, Information Management and Clinical Systems, NSW Department of Health by phone at (02) 93929110 , or by email at pwill@doh.health.nsw.gov.au.

\section{QUALITY OF REPORTING OF ABORIGINALITY TO THE NSW MIDWIVES DATA COLLECTION}

\section{Lee Taylor and Kim Lim}

Epidemiology and Surveillance Branch NSW Department of Health

This article describes a study that used capture-recapture methods to assess the quality of information on Aboriginality reported to the NSW Midwives Data Collection (MDC).

The NSW Aboriginal Health Strategic Plan states that: 'In order to measure improvements and effectively target funding to programs which will improve the health of Aboriginal people, strategies are required to develop robust performance indicators, improve data collection and improve reporting processes'. ${ }^{1}$ Aboriginality is known to be under-reported on Department of Health data collections in NSW, although it is not known to what extent. Improving the quality of information on Aboriginality in health data collections is an important part of improving the overall quality of information on Aboriginal health in NSW.

The MDC is a population-based surveillance system covering all births in NSW public and private hospitals, as well as homebirths. Births in NSW are required to be reported to the MDC under the NSW Public Health Act
1991. The data are used to monitor trends and variations in mortality and morbidity of mothers and newborns, quality of care and the major risk factors for adverse outcomes for mothers and babies. The MDC encompasses all live births and stillbirths of at least 20 weeks gestation or at least 400 grams birth-weight.

\section{METHODS}

The Aboriginality of the mother, rather than the baby, is reported to the MDC, although mother's Aboriginality is frequently used as a proxy measure for the baby's Aboriginality. Consequently, maternal Aboriginality was used for this analysis.

Aboriginal or Torres Straight Islander mothers were counted as one group in the MDC up to 1997 and as two separate groups thereafter. We were therefore unable to examine trends in the quality of reporting for both these groups. For ease of reference, in this report 'Aboriginal' will be used to refer to both groups combined.

Records of births reported to the MDC were linked to birth registration records of the NSW Registry of Births, Deaths and Marriages for births occurring in the five-year period 1994-98. Records from the two files were matched using a probabilistic linkage software (Automatch). Prior 